\title{
High-Confinement, High-Q Microring Resonators on Silicon Carbide-On-Insulator (SiCOI)
}

Zheng, Yi; Yi, Ailun; Pu, Minhao; Chang, Bingdong; You, Tiangui; Huang, Kai; Ou, Xin; Ou, Haiyan

\section{Published in:}

Proceedings of Asia Communications and Photonics Conference

Link to article, DOI:

10.1109/ACP.2018.8595751

Publication date:

2018

Document Version

Peer reviewed version

Link back to DTU Orbit

Citation $(A P A)$ :

Zheng, Y., Yi, A., Pu, M., Chang, B., You, T., Huang, K., Ou, X., \& Ou, H. (2018). High-Confinement, High-Q Microring Resonators on Silicon Carbide-On-Insulator (SiCOI). In Proceedings of Asia Communications and Photonics Conference IEEE. https://doi.org/10.1109/ACP.2018.8595751

\section{General rights}

Copyright and moral rights for the publications made accessible in the public portal are retained by the authors and/or other copyright owners and it is a condition of accessing publications that users recognise and abide by the legal requirements associated with these rights.

- Users may download and print one copy of any publication from the public portal for the purpose of private study or research.

- You may not further distribute the material or use it for any profit-making activity or commercial gain

- You may freely distribute the URL identifying the publication in the public portal 


\section{High-Confinement, High- $Q$ Microring Resonators on Silicon Carbide-On-Insulator (SiCOI)}

\author{
Yi Zheng ${ }^{\dagger}$ \\ DTU Fotonik, Technical University of \\ Denmark \\ Lyngby, Denmark \\ yizhen@fotonik.dtu.dk \\ Bingdong Chang \\ DTU Danchip, National Center for \\ Micro- and Nanofabrication, Technical \\ University of Denmark \\ Lyngby, Denmark \\ bincha@dtu.dk \\ $\mathrm{Xin} \mathrm{Ou}$ \\ Shanghai Institute of Microsystem and \\ Information Technology, Chinese \\ Academy of Sciences \\ Shanghai, China \\ ouxin@mail.sim.ac.cn
}

\author{
Ailun $\mathrm{Yi}^{\dagger}$ \\ Shanghai Institute of Microsystem and \\ Information Technology, Chinese \\ Academy of Sciences \\ Shanghai, China \\ alyi@mail.sim.ac.cn
}

Tiangui You

Shanghai Institute of Microsystem and

Information Technology, Chinese

Academy of Sciences

Shanghai, China

t.you@mail.sim.ac.cn

Haiyan $\mathrm{Ou}^{*}$

DTU Fotonik, Technical University of

Denmark

Lyngby, Denmark

*haou@fotonik.dtu.dk

\author{
Minhao Pu \\ DTU Fotonik, Technical University of \\ Denmark \\ Lyngby, Denmark \\ mipu@fotonik.dtu.dk
}

\author{
Kai Huang \\ Shanghai Institute of Microsystem and \\ Information Technology, Chinese \\ Academy of Sciences \\ Shanghai, China \\ khuang@mail.sim.ac.cn
}

\begin{abstract}
We realize silicon carbide-on-insulator platform based on crystalline $4 \mathrm{H} \mathrm{SiC}$ and demonstrate high confinement $\mathrm{SiC}$ resonators with sub-micron waveguide cross-sectional dimension. The obtained $Q(57,000)$ is the highest $Q$ reported for $\mathrm{SiC}$ microring resonators.
\end{abstract}

Keywords-(230.0230) Optical devices; (230.5750) Resonators; (190.4390) Nonlinear optics, integrated optics.

\section{INTRODUCTION}

Tremendous effort has been made in the last decade to develop integrated nonlinear platforms using different materials including silica [1], silicon [2], $\mathrm{Si}_{3} \mathrm{~N}_{4}$ [3], Hydex [4], diamond [5], AlN [6], and AlGaAs [7]. Silicon Carbide (SiC) is also a promising material for nonlinear applications thanks to its high material nonlinearities. $\mathrm{SiC}$ has a refractive index of 2.6 around

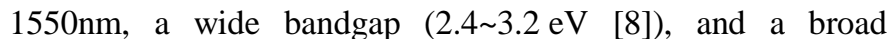
transparent window $\left(0.37 \sim 5.6 \mu \mathrm{m}\right.$ [9]). Its quadratic $\left(\chi^{(2)}\right)$ nonlinearity is $\sim 30 \mathrm{pm} / \mathrm{V}$, which is comparable to that of LiNbO3 [10], while its Kerr $\left(\chi^{(3)}\right)$ nonlinearity is on the order of $10^{-18} \mathrm{~m}^{2} \mathrm{~W}^{-1}$ [11], which is one order of magnitude larger than $\mathrm{Si}_{3} \mathrm{~N}_{4}$ [3]. Moreover, point defects in $\mathrm{SiC}$ are being exploited for single-photon sources for quantum applications [12], [13]. $\mathrm{SiC}$ photonic devices including photonic crystal, microdisk, and microring resonators have been studied for many years, and different applications have been demonstrated like second harmonic generation [14], parametric frequency conversion [15], self-phase modulation [16], and single-photon generation [17]. For nonlinear applications, not only high quality factor $(Q)$ resonators are critical components for efficient nonlinear applications due to an intra-cavity field enhancement, but high confinement waveguides are also desirable due to an effective nonlinearity enhancement. To realize integrated highconfinement waveguides in $\mathrm{SiC}$, a SiC thin film of high quality should be fabricated with a low-index cladding (e.g. air or glass). Among more than 200 polytypes of different crystalline

This work was supported by the DNRF Research Centre of Excellence, SPOC (DNRF123) and the National Natural Science Foundation of China (No.: 11705262, 11622545 and U1732268). ), Frontier Science Key Program of CAS (No.: QYZDY-SSW-JSC032), One Hundred Talent Program of CAS, International Collaboration Project of Shanghai (No.: 16520721100). ${ }^{\dagger}$ These authors contributed equally to this work.
SiC materials, 3C-, $4 \mathrm{H}-$ and $6 \mathrm{H}-\mathrm{SiC}$ are the most common polytypes, which are commercially available. Such high confinement $3 \mathrm{C}$-SiC photonic devices can be readily fabricated as $3 \mathrm{C}-\mathrm{SiC}$ is available commercially in epitaxially grown films on silicon substrates. However, the growth-induced stacking defects result in a high material absorption loss. The highest $\mathrm{Q}$ demonstrated for $3 \mathrm{C}-\mathrm{SiC}$ microring resonators is $\sim 42,000$ [18]. $4 \mathrm{H}-$ and $6 \mathrm{H}-\mathrm{SiC}$ offer a higher crystal quality but are only available in bulk crystalline wafers. Smart-cut, a mature technology for silicon-on-insulator wafer fabrications [19], can be utilized to fabricate silicon carbide-on-insulator (SiCOI) wafers [20]. However, large dimension waveguides have been used in previous demonstrations [16],[21] to mitigate the scattering loss from rough waveguide sidewalls at the price of reduced effective nonlinearities. In this paper, we fabricate high-confinement $4 \mathrm{H}-\mathrm{SiCOI}$ microring resonators using optimized patterning processes and demonstrate the highest $\mathrm{Q}$ $(\sim 57,000)$ for $\mathrm{SiC}$ microring resonators.

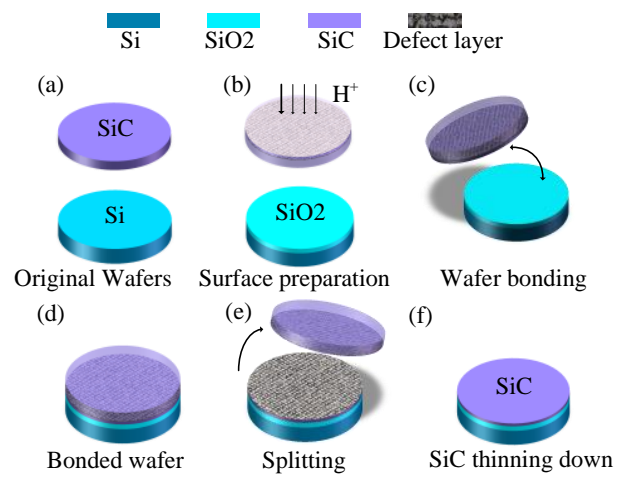

Fig. 1. (a) $\mathrm{SiC}$ and silicon carrier wafer. (b) $\mathrm{H}+$ ion implantation into bulk crystaline $\mathrm{SiC}$ wafer and thermal oxidation of silicon carrier wafer. (c) Wafer bonding between thermal oxidized silicon wafer and ion implantated $\mathrm{SiC}$ wafer. (d) Bonded wafer. (e) Splitting of $\mathrm{SiC}$ wafer after high temperature annealing and (f) SiCOI wafer after SiC layer thinning down. 


\section{FABRICATION}

The fabrication process of the SiCOI wafer is shown in Figure 1. First, the $4 \mathrm{H}$ bulk $\mathrm{SiC}$ wafer was implanted with $170 \mathrm{keV} \mathrm{H}^{+}$species. $\mathrm{H}^{+}$peak concentration was simulated to be at $1.1 \mu \mathrm{m}$ under the wafer surface and micro-cavities (defects) are created along the peak concentration [22]. The silicon carrier wafer was thermally oxidized to grow a $2-\mu \mathrm{m}$ thick silicon dioxide layer. After cleaning of both wafers, they were bonded together by using direct wafer bonding. The bonded wafers were then annealed at around $850^{\circ} \mathrm{C}$ to split the thin $\mathrm{SiC}$ film from the bulk SiC wafer. The last step was thinning down of $\mathrm{SiC}$ layer. A common method is chemical mechanical polishing [23], which can remove defective $\mathrm{SiC}$ material and smooth the surface. Here, the $\mathrm{SiC}$ was thinned down from $1.1 \mu \mathrm{m}$ to $500 \mathrm{~nm}$.
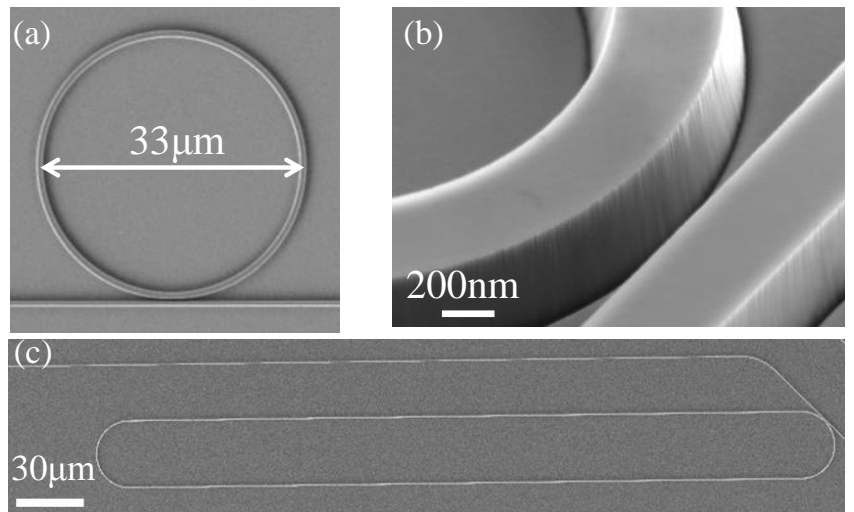

Fig. 2. Scanning electron microscopy (SEM) picttures of a fabricated microring resonator with a radius of $16.5 \mu \mathrm{m}$ (a) and its coupling region (b). (c) A 1004- $\mu \mathrm{m}$ long racetrack ring resonator.

The device fabrication on the SiCOI wafer started from electron beam lithography. A 600-nm thick electron-beam resist layer (hydrogen silsesquioxane) was spun on SiCOI wafer as the etching mask. The device pattern was transferred to the $\mathrm{SiC}$ layer in an inductively coupled plasma reactive ion etching machine using fluorine-based gases $\left(\mathrm{SF}_{6}\right)$. Figure 2(a) shows a $16.5-\mu \mathrm{m}$ radius $(1 \mathrm{THz})$ microring resonator and figure 2(b) shows the coupling region of the microring resonator with a coupling gap of $300 \mathrm{~nm}$. Figure 2(c) is a 1004$\mu \mathrm{m}$ long raceteack ring resonator.
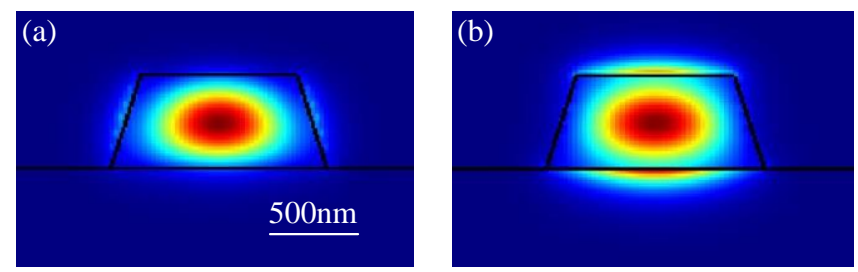

Fig. 3. Simulated mode profile for the fundamental TE (a) and TM (b) modes with effective areas of $0.32 \mu \mathrm{m}^{2}$ and $0.30 \mu \mathrm{m}^{2}$.

The fabricated $\mathrm{SiC}$ waveguides are $500 \mathrm{~nm}$ high and $680 \mathrm{~nm}$ wide, and the mode profiles for fundamental transverse electric (TE) and transverse magnetic (TM) modes are shown in figure 3(a) and 3(b), respectively. Since the effective nonlinearity $\gamma$ is highly dependent on the effective mode area $A_{\text {eff }}$ as expressed by the equation $\gamma=2 \pi n_{2} / \lambda A_{\text {eff }}$, where $n_{2}$ is the nonlinear refractive index of $\mathrm{SiC}$ [11] and $\lambda$ is the operating wavelength. Thanks to the strong light confinement in such a sub-micron dimension waveguide, a large effective nonlinearity at the order of $10 \mathrm{~W}^{-1} \mathrm{~m}^{-1}$ can be expected.

\section{CHARACTERIZATION}

To characterize the performance of SiCOI devices, we fabricated straight waveguides with different lengths and microring resonators including $16.5-\mu \mathrm{m}$ radius microring resonators and $1004-\mu \mathrm{m}$ long racetrack resonators. Figure 4 shows the measured insertion losses of waveguides with different lengths for fundamental TE and TM modes. The estimated linear losses for straight waveguides are $10 \mathrm{~dB} / \mathrm{cm}$ and $9.3 \mathrm{~dB} / \mathrm{cm}$ for TE and TM modes, respectively.
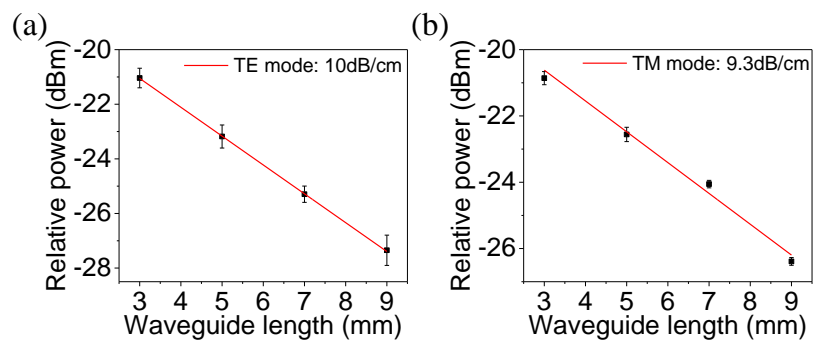

Fig. 4. Measured insertion loss of SiCOI waveguides with different lengths for the fundamental TE (a) and TM mode (b).

Figure5 shows the normalized transmission spectra of $16.5-\mu \mathrm{m}$ radius microring resonator (figure $5(\mathrm{a}, \mathrm{b}, \mathrm{e}, \mathrm{f})$ ) and $1004-\mu \mathrm{m}$ long racetrack ring resonator (figure $5(\mathrm{c}, \mathrm{d}, \mathrm{g}, \mathrm{h}))$. The resonances were fitted with Lorentzian curve. As those tested devices work in under-coupling region, the intrinsic $\mathrm{Q}$ factor can be calculated as $Q_{\text {int }}=2 Q_{\text {load }} /(1+\sqrt{T})$ [25], where $Q_{\text {load }}$ is the loaded $\mathrm{Q}$ and $\mathrm{T}$ is the transmission normalized to the maximum value of fittig curve. The free spectrum range (FSR) was measured as $1.008 \mathrm{THz}$ and $0.956 \mathrm{THz}$ for fundamental TE and TM modes in 16.5- $\mu \mathrm{m}$ radius microring resonators, respectively. $103.8 \mathrm{GHz}$ and $98.8 \mathrm{GHz}$ FSR were measured for TE and TM modes in 1004- $\mu$ m long racetrack ring resonators, respectively. We achieve intrinsic $Q$ factor of 50,000 and 51,000 for fundamental TE modes in $16.5-\mu \mathrm{m}$ radius microring resonator and $1004-\mu \mathrm{m}$ long racetrack ring resonator, respectively. Q factor of 48,000 and 57,000 are obtained for fundamental TM modes in $16.5-\mu \mathrm{m}$ radius microring resonator and $1004-\mu \mathrm{m}$ long racetrack ring resonator, respetively. The achieved 57,000 is the highest $\mathrm{Q}$ factor reported in crystalline $\mathrm{SiC}$ microring resonators. Since the $1004-\mu \mathrm{m}$ long racetrack ring resonator consists of $16.5-\mu \mathrm{m}$ radius curved waveguide and $900 \mu \mathrm{m}$ straight waveguide, we also extract the linear loss of straight waveguide part for TE and TM modes and $9.8 \mathrm{~dB} / \mathrm{cm}$ and $9 \mathrm{~dB} / \mathrm{cm}$ were obtained, respectively. The extracted linear loss for straight waveguides matches quite well with measured straight waveguide loss, which confirms the measurement results of straight waveguides.

\section{CONCLUSION}

High confinement waveguides with sub-micron crosssectional dimensions $\left(500 \times 680 \mathrm{~nm}^{2}\right)$ and microring resonators in the SiCOI platform was demonstrated. a high $Q$ of 57,000 for a $1004-\mu \mathrm{m}$ racetrack resonator was obtained, which is the highest reported $Q$ for crystalline $\mathrm{SiC}$ microring resonators. 
The demonstrated high $Q$, high confinement microresonators are promising in nonlinear applications, such as frequency comb generation. To improve the performance of SiCOI devices, the dry-etching process can be optimized to reduce sidewall roughness-induced scattering. Furthermore, the crystalline quality of $\mathrm{SiC}$ thin fiim can be improved by $\mathrm{H}^{+}$ implantation at elevated temperature [25]or post annealing at high temperature .

(a)

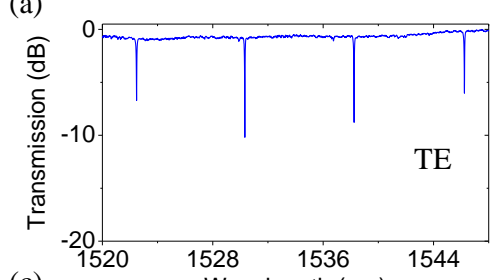

(c)
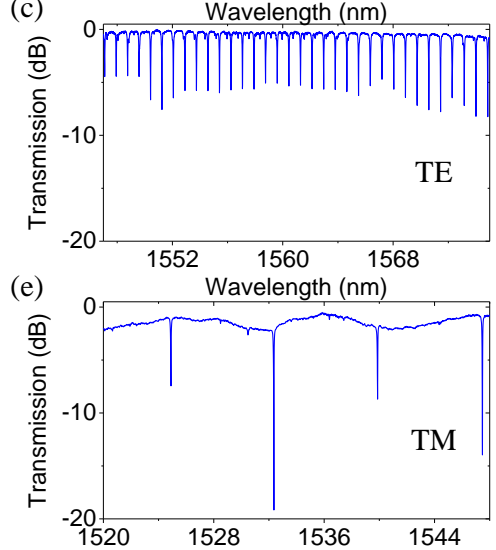

(g)

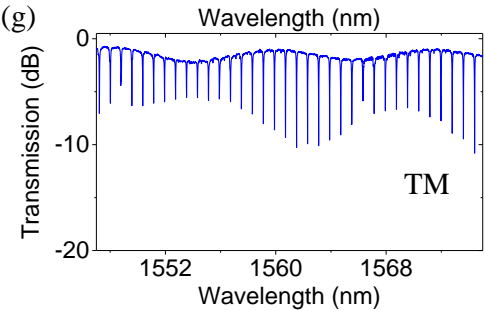

(b)

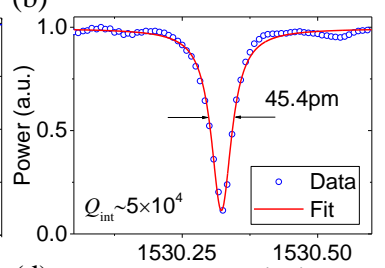

(d)

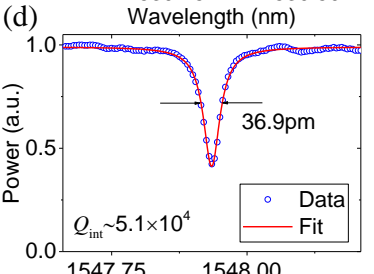

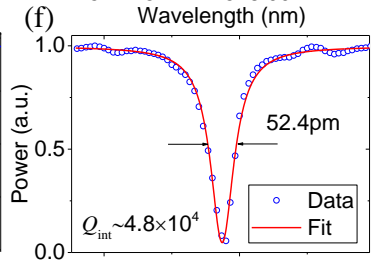

(h) ${ }^{1547.25}$ Wavelength $(\mathrm{nm})$

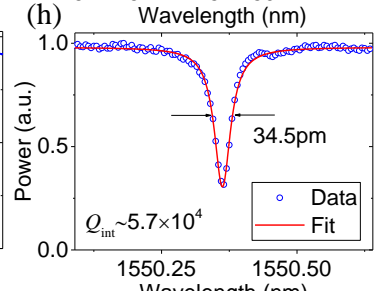

Fig. 5. Measured (normalized) transmission spectra for $16.5-\mu \mathrm{m}$ radius microring resonators $(\mathrm{a}, \mathrm{b}, \mathrm{e}, \mathrm{f})$ and $1004-\mu \mathrm{m}$ long racetrack resonators $(\mathrm{c}, \mathrm{d}, \mathrm{g}$, h) for the fundamental TE mode (a-d) and TM mode (e-h).

\section{REFERENCES}

[1] K. Y. Yang et al., "Bridging ultrahigh-Q devices and photonic circuits," Nat. Photonics, vol. 12, no. 5, pp. 297-302, 2018.

[2] A. G. Griffith et al., "Silicon-Chip Mid-Infrared Frequency Comb Generation," Nat. Commun., vol. 6, 2014.

[3] J. S. Levy, A. Gondarenko, M. A. Foster, A. C. Turner-Foster, A. L. Gaeta, and M. Lipson, "CMOS-compatible multiple-wavelength oscillator for on-chip optical interconnects," Nat. Photonics, vol. 4, no. 1 , pp. 37-40, 2010.

[4] L. Razzari et al., "CMOS-compatible integrated optical hyperparametric oscillator," Nat. Photonics, vol. 4, no. 1, pp. 41-45, 2010.

[5] B. J. M. Hausmann, I. Bulu, V. Venkataraman, P. Deotare, and M. Lončar, "Diamond nonlinear photonics," Nat. Photonics, vol. 8, no. 5, pp. 369-374, 2014.

[6] H. Jung, C. Xiong, K. Y. Fong, X. Zhang, and H. X. Tang, "Optical frequency comb generation from aluminum nitride microring resonator," Opt. Lett., vol. 38, no. 15, p. 2810, 2013.

[7] M. Pu, L. Ottaviano, E. Semenova, and K. Yvind, "Efficient frequency comb generation in AlGaAs-on-insulator," Optica, vol. 3, no. 8, pp. 823-826, 2016.

[8] V. E. Chelnokov and A. L. Syrkin, "High temperature electronics uising $\mathrm{SiC}$ : actual situation and unsolved problems," Materials Science and Engineering, vol. 46. pp. 248-253, 1997.

[9] S. Wang et al., "4H-SiC: A new nonlinear material for midinfrared lasers," Laser Photonics Rev., vol. 7, no. 5, pp. 831-838, 2013.

[10] I. J. Wu and G. Y. Guo, "Second-harmonic generation and linear electro-optical coefficients of SiC polytypes and nanotubes," Phys. Rev. B - Condens. Matter Mater. Phys., vol. 78, no. 3, 2008.

[11] F. De Leonardis, R. A. Soref, and V. M. N. Passaro, "Dispersion of nonresonant third-order nonlinearities in Silicon Carbide," Sci. Rep., vol. 7, p. 40924, 2017.

[12] S. Castelletto et al., "A silicon carbide room-temperature singlephoton source," Nat. Mater., vol. 12, 2013.

[13] D. Christle, A. Falk, and P. Andrich, "Isolated electron spins in silicon carbide with millisecond-coherence times," Nat. Mater., vol. 14, no. February, pp. 160-163, 2014.

[14] S. Yamada et al., "Second-harmonic generation in a silicon-carbidebased photonic crystal nanocavity.," Opt. Lett., vol. 39, no. 7, pp. 1768-71, 2014

[15] J. Cardenas et al., "Parametric Frequency Conversion in Silicon Carbide Waveguides," in CLEO, 2015, p. SF1D.7.

[16] J. Cardenas et al., "Optical nonlinearities in high-confinement silicon carbide waveguides," Opt. Lett., vol. 40, no. 17, pp. 4138 4141, 2015.

[17] M. Widmann et al., "Coherent control of single spins in silicon carbide at room temperature," Nat. Mater., vol. 14, no. 2, pp. 164 168, 2014.

[18] T. Fan, H. Moradinejad, X. Wu, A. A. Eftekhar, and A. Adibi, "High Q Integrated Photonic Microresonators on 3C SiC-onInsulator Platform," CLEO, 2018.

[19] J. Pan, J. Cooper, M. Melloch, S. material, and M. Bruel, "Silicon on insulator material technology," IEEE Electronic letter, vol. 75, no. 31, pp. 41-3205, 1994.

[20] L. Di Cioccio, F. Letertre, Y. Le Tiec, A. M. Papon, C. Jaussaud, and M. Bruel, "Silicon carbide on insulator formation by the SmartCut@process," Mater. Sci.Eng. B46,pp.349-356, 1997.

[21] J. Cardenas et al., "Optical Nonlinearities in High Confinement SiC Waveguides," in CLEO: 2014, 2014, p. SW3I.4.

[22] Q.-Y. Tong et al., "Layer splitting process in hydrogen-implanted $\mathrm{Si}, \mathrm{Ge}, \mathrm{SiC}$, and diamond substrates Hydrogen ion implantation mechanism in GaAs-on-insulator wafer formation by ion-cut process Layer splitting process in hydrogen-implanted $\mathrm{Si}, \mathrm{Ge}, \mathrm{SiC}$, and diamond substrates," Appl. Phys. Lett., vol. 70, no. 10, pp. 13901138, 1997.

[23] L. Zhou, "Chemomechanical Polishing of Silicon Carbide," J. Electrochem. Soc., vol. 144, no. 6, p. L161, 1997.

[24] P. E. Barclay, K. Srinivasan, and O. Painter, "Nonlinear response of silicon photonic crystal microresonators excited via an integrated waveguide and fiber taper," Opt. Express, vol. 13, pp. 801-820, 2005.

[25] R. B. Gregory, T. A. Wetteroth, S. R. Wilson, O. W. Holland, and D. K. Thomas, "Effects of irradiation temperature and dose on exfoliation of implanted silicon carbide Effects of irradiation temperature and dose on exfoliation of $\mathrm{H}$-implanted silicon carbide," Appl. Phys. Lett., vol. 75, no. 982, pp. 2623-1390, 1999. 